\title{
International Conference on Smart Materials and Surfaces (SMS), Bangkok
}

\section{Dear Reader,}

We are pleased to announce International Conference on Smart Materials and Surfaces (SMS). It will be organised jointly by SETCOR, IAAM and VBRI Press during 26-28 August 2014 at Sheraton Grande Sukhumvit Hotel, Bangkok, Thailand. Smart Materials and Surfaces (SMS), Bangkok is a three-day event targeting researchers interested in the design, modification, characterisation and applications of Novel Smart \& Active Surfaces and Materials. The goal of conference is to provide a global platform for researchers and engineers coming from academia and industry to present their research results and activities in the field of Intelligent Materials science and engineering. The conference will provide opportunities for the delegates to exchange face-to-face their novel ideas and experiences with the international experts during plenary \& invited talks, oral presentations and poster sessions. We will set up sessions with keynote forum, panel discussion, project negotiation along with welcome cocktail and gala dinner.

\section{General Chairs}

\section{Prof. Ashutosh Tiwari}

Biosensors and Bioelectronics Centre

Linköping University, Sweden

\section{Dr. Naveed Anwar}

Executive Director/CEO

AIT Consulting Affiliated Faculty

Structural Engineering Director, ACECOMS

Asian Institute of Technology, Thailand

\section{Conference Themes}

The Smart Materials \& Surfaces conference represents a powerful and unique opportunity to interact with a range of researchers all interested in creating and applying modified surfaces and materials. Sessions will include (but not restricted to):

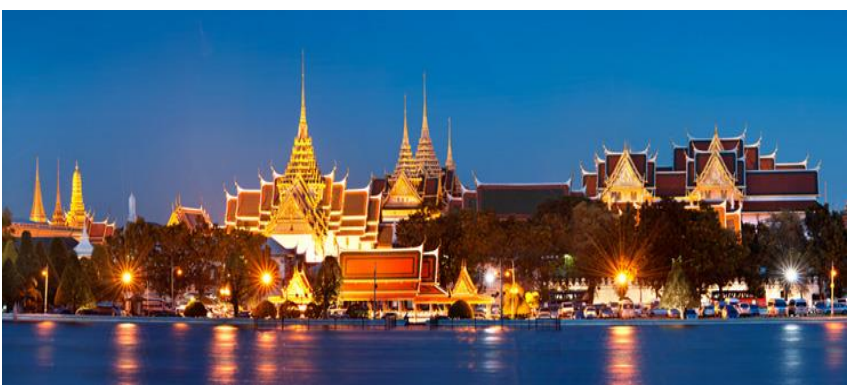

Image of SMS, Bangkok venue
Development and Characterization of Multifunctional and Smart Materials/Surfaces/Coatings

- Material/coating design, formulation, processing and characterisation.

- Design and Theory of Smart Surfaces

- New manufacturing technologies

- Interface and interaction science

- Hybrid/ composite materials design and optimisation

- Biological and bio-inspired composite materials/coatings

- Novel nano and microdevices

- Nanocomposite, Bionanocomposite, etc

- Nanostructured, nanoporous, etc

- Piezoelectric, ferroelectric, electroactive, electromagnetic, self-healing, heating, sensing materials.

\section{Characterisation/Properties of Active Materials/} Surfaces/ Coatings

- Micro- and nano-characterization of mechanical and electromechanical properties

- Damage, fatigue, aging, and fracture mechanics of smart devices

- Design optimization

- Mechanics of nano-devices

- Continuum models for nonlinear multi-field coupled behavior

- Micromechanical and thermodynamical modeling approaches

- Nano-scale effects and atomistic modeling techniques

- Advanced mathematical methods, finite element formulations and implementation

- Modeling, Simulation and Control of Adaptive Systems

- Integrated System Design and Implementation

- $\quad$ Structural Health Monitoring

\section{Smart Materials/ Coatings Applications}

- Energy harvesting and storage

- MEMS and NEMS devices and applications

- Biomedical, Prosthetic and Implant Materials, Biosensors,

- Food and pharmaceutical packaging

- Automotive, aerospace, textile, construction, etc

We trust that Advanced Materials Letters offers an international interactive platform for both industrial and academic researchers, professors and students active in the fields of applied or fundamental materials science related to biological, chemical, earth, atmosphere, ocean and planetary, engineering, mathematical, medical and physical 
sciences. The journal is expected to become popular accompanied with a high impact factor and as a conference chair, may welcome you as active part of the SMS, Bangkok. Your opinions and suggestions are greatly valued for the success of this event.
You may follow

https://www.facebook.com/SETCOR

With kindest regards,

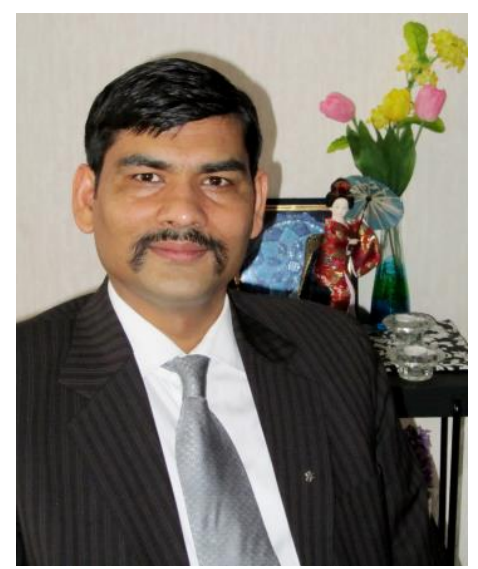

\section{Ashutosh Tiwari, PhD, DSc}

Conference Chair

International Conference on Smart Materials and Surfaces, Bangkok, Thailand

Editor-in-Chief, Advanced Materials Letters

Biosensors \& Bioelectronics Centre

IFM-Linköping University, Sweden 